\title{
Multi-scale Recordings for Neuroprosthetic Control of Finger Movements
}

\author{
Justin Baker, William Bishop, Spencer Kellis, Todd Levy, Paul House MD, and Bradley Greger PhD
}

\begin{abstract}
We trained a rhesus monkey to perform individuated and combined finger flexions and extensions of the thumb, index, and middle finger. A Utah Electrode Array (UEA) was implanted into the hand region of the motor cortex contralateral to the monkey's trained hand. We also implanted a microwire electrocorticography grid ( $\mu$ ECoG) epidurally so that it covered the UEA. The $\mu$ ECoG grid spanned the arm and hand regions of both the primary motor and somatosensory cortices. Previously this monkey had Implantable MyoElectric Sensors (IMES) surgically implanted into the finger muscles of the monkey's forearm. Action potentials (APs), local field potentials (LFPs), and $\mu$ ECoG signals were recorded from wired head-stage connectors for the UEA and $\mu$ ECoG grids, while EMG was recorded wirelessly. The monkey performed a finger flexion/extension task while neural and EMG data were acquired. We wrote an algorithm that uses the spike data from the UEA to perform a real-time decode of the monkey's finger movements. Also, analyses of the LFP and $\mu$ ECoG data indicate that these data show trial-averaged differences between different finger movements, indicating the data are potentially decodeable.
\end{abstract}

\section{INTRODUCTION}

As the field of neuroprosthetics matures, researchers are attempting to access both muscular as well as neural signals to use as control signals in the least invasive way possible. Neural and muscular recording devices are moving toward wireless technologies in order to avoid the infection risks associated with percutaneous connectors. Likewise, control signals can be accessed at different sites, e.g. residual muscle, peripheral nerve, epidural, subdural, or intracortical. Implementations of neural prosthetics should always be biased towards the least invasive method that effectively restores lost function. However, more complex devices that access neural and myoelectric signals at multiple levels may provide sufficient improvement in prosthetic control to justify more invasive devices. In order to gain empirical data relevant to these issues,

Manuscript received April 23, 2009. This work was supported in part by DARPA BAA05-26 Revolutionizing Prosthetics.

J. Baker is a bioengineering graduate student at the University of Utah, Salt Lake City, UT, 84112 USA (e-mail: justin.j.baker@utah.edu).

W. Bishop works at the Applied Physics Laboratory at Johns Hopkins University, 11100 Johns Hopkins Rd, Laurel, MD, 20723 USA (e-mail: William.Bishop@jhuapl.edu).

S. Kellis is a graduate student at the University of Utah, Salt Lake City, UT 84112 USA (e-mail: spencer.kellis@utah.edu).

T. Levy works at the Applied Physics Laboratory at Johns Hopkins University, 11100 Johns Hopkins Rd, Laurel, MD, 20723 USA (e-mail: Todd.Levy@jhuapl.edu).

P. House is a board certified neurosurgeon at the University of Utah, Salt Lake City, UT, 84112 USA (e-mail: paul.house@hsc.utah.edu).

B. Greger is an assistant professor with the Bioengineering Department at the University of Utah, Salt Lake City, UT 84112 USA (e-mail: bradley.greger@utah.edu). we implanted three recording devices into sites with different degrees of invasiveness for accessing neural and myoelectric signals. Using these devices, we have begun examining the ability of using AP, LFP, and epidural $\mu \mathrm{ECoG}$ data to write algorithms capable of decoding individual and combined finger movements.

\section{METHODS}

\section{The Behavioral Task}

A male monkey (Macaca mulatta) was trained to perform cued flexions of the thumb, flexions and extensions of the index finger and middle finger, and combined flexions involving different combinations of flexions of the thumb, index finger, and middle finger using a manipulandum which uses microswitches to monitor finger flexions and extensions [1]. In full, the monkey was trained to perform nine different movements. (see Fig. 1).

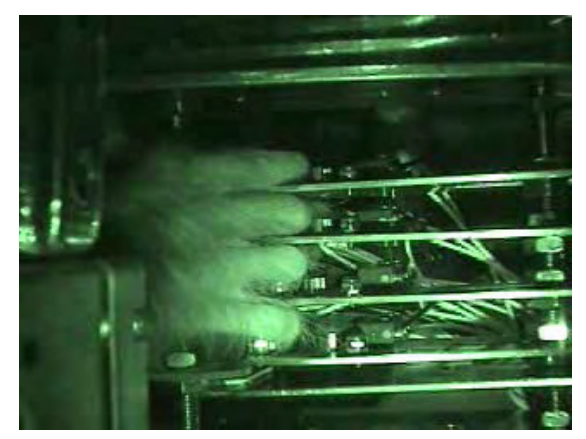

Fig. 1. Infrared photograph of the monkey's right hand in the manipulandum.

The behavioral finger task was programmed using LabVIEW (National Instruments) and ran on a real-time embedded computer. A computer screen placed in front of the monkey was used to visually cue the desired finger movement. The first state in the behavioral task requires that the monkey relax all his fingers so that none of the finger switches are pressed (see Fig. 2). The monkey then waited for the finger movement cue for a randomized time between 1000-3000 ms. At the end of this wait period the monkey received a visual cue indicating which finger it should flex/extend. The monkey then had $2000 \mathrm{~ms}$ in which to flex/extend the cued finger(s) and depress the associated microswitch. The monkey was required to keep the microswitch pressed for a $500 \mathrm{~ms}$ hold time. If the monkey flexed the correct finger and did not violate the timing constraints, it received a juice reward [2]. 


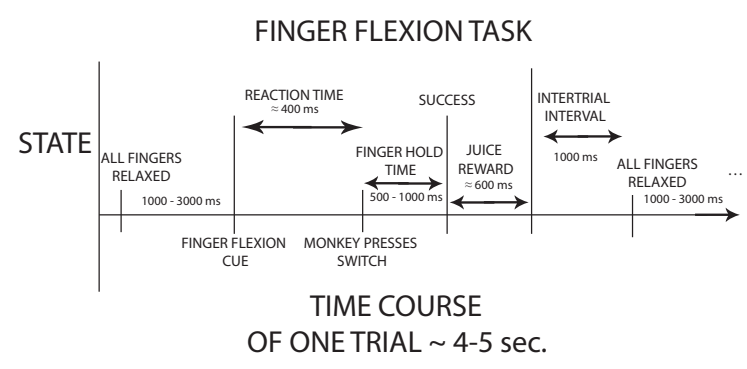

Fig. 2. Time course and states during the finger flexion behavioral task.

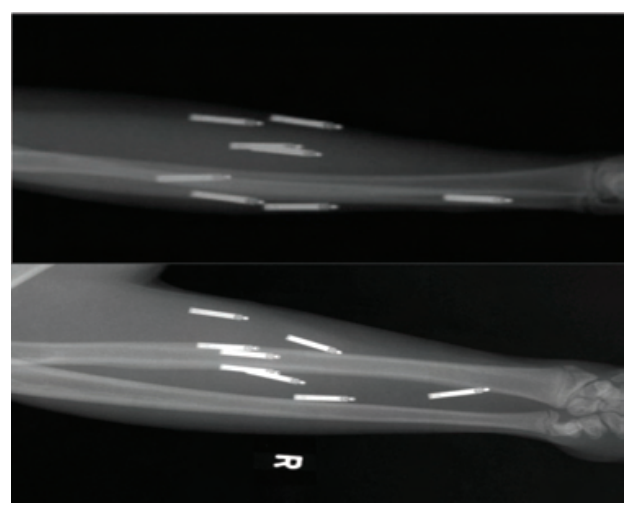

Fig. 3. (Top) Radial view x-ray of eight IMES implanted into the monkey's right arm taken 1 week post-implantation. (Middle) Dorsal view x-ray of implanted IMES taken 19 months post-implantation. Note that after 19 months the IMES remain positionally stable and did not migrate within the muscles.

\section{UEA, $\mu E C o G$, and IMES}

The UEA is a $10 \times 10$ array of $1 \mathrm{~mm}$ long microelectrodes with 400 micron inter-electrode spacing [3]. The UEA has 96 recording electrodes and records single-unit and multi-unit action potentials as well as LFP. We implanted a UEA intracortically into the hand region of the motor cortex contralateral to the monkey's trained hand (see Fig. 4). A $2 \times 2 \mathrm{~cm} 96$ channel $\mu \mathrm{ECoG}$ grid with $1 \mathrm{~mm}$ inter-electrode spacing was implanted in the epidural space. The $\mu \mathrm{ECoG}$ grid was implanted during the same surgery as the UEA, and was placed above the UEA so that it covered the arm and hand regions of primary motor and somatosensory cortex (see Fig. 4). Each IMES is approximately $2 \mathrm{~mm}$ in diameter and $15 \mathrm{~mm}$ long, with active electrodes on each end [4]-[6]. The IMES use inductive coupling to provide power and transmit the data [7]. In an earlier surgery we surgically implanted IMES into different finger and wrist flexion and extension muscles of the monkey's right forearm (see Fig. 3) [2].

\section{Instrumentation}

A typical recording session involves placing the monkey in a primate chair and then placing the monkey into a shielded Faraday chamber in front of a video screen used to cue the monkey. Two Cerebus systems (Blackrock Microsystems, Salt Lake City, UT) with synchronized clocks, providing a total of 256 channels, were used to acquire the UEA, $\mu \mathrm{ECoG}$, and IMES data; as well as the monkey's behavioral data. Recording

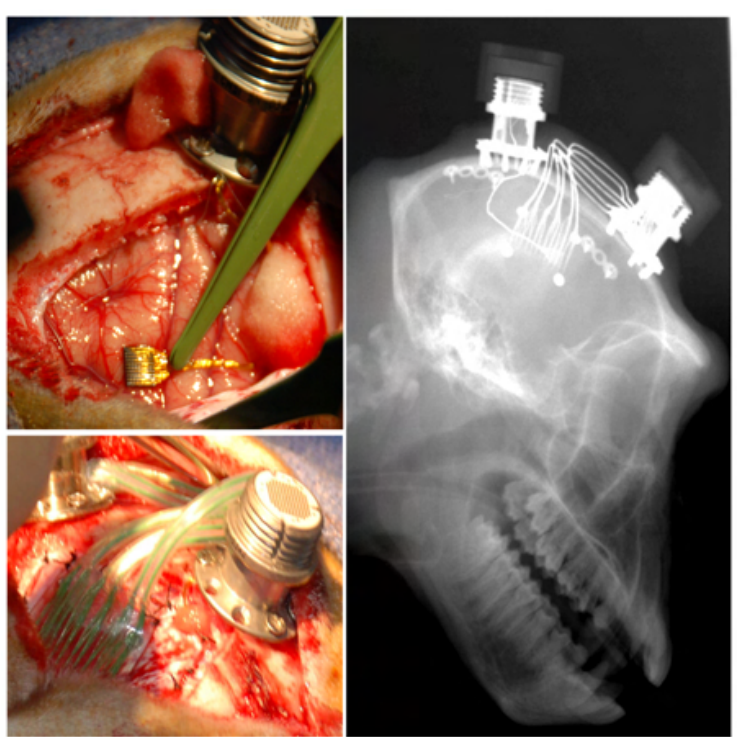

Fig. 4. (Top left) UEA implanted into hand region of primary motor cortex. (Bottom left) Microwire ECoG grid implanted epidurally over the hand and arm region of primary motor and somatosensory cortex. Note the difference in scale between the $\mu \mathrm{ECoG}$ grid and the UEA. (Right) X-ray taken 4 months post implantation showing stability of device locations.

sessions resulted in approximately 30 minutes to 2 hours of data. The IMES EMG data were sampled at $1.26 \mathrm{kHz}$, the UEA data were sampled at $30 \mathrm{kHz}$, and the ECoG data were sampled at $1 \mathrm{kHz}$. The behavioral task interfaced with the data acquisition system using a PXI-digital/analog IO card. The user interface for controlling the experiment was provided via a second computer which communicated with the real-time embedded computer over Ethernet.

\section{RESULTS}

\section{Individual Unit Tuning Curves}

Neural data recorded from the UEA were sorted offline using Offline Sorter v2.8.7 (Plexon). The individual units were then exported into MATLAB for analysis. Action potential times in each unit were windowed and then aligned around the

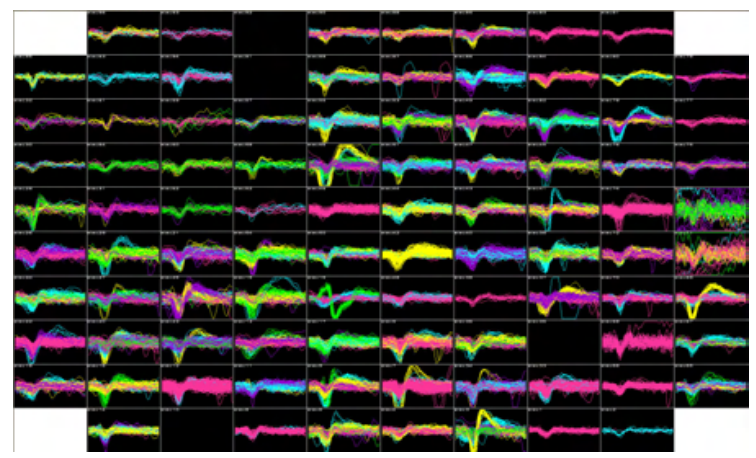

Fig. 5. Spike panel showing online sorted action potentials across the UEA as the monkey performs the finger task. Note the quality and number of APs across the array. 


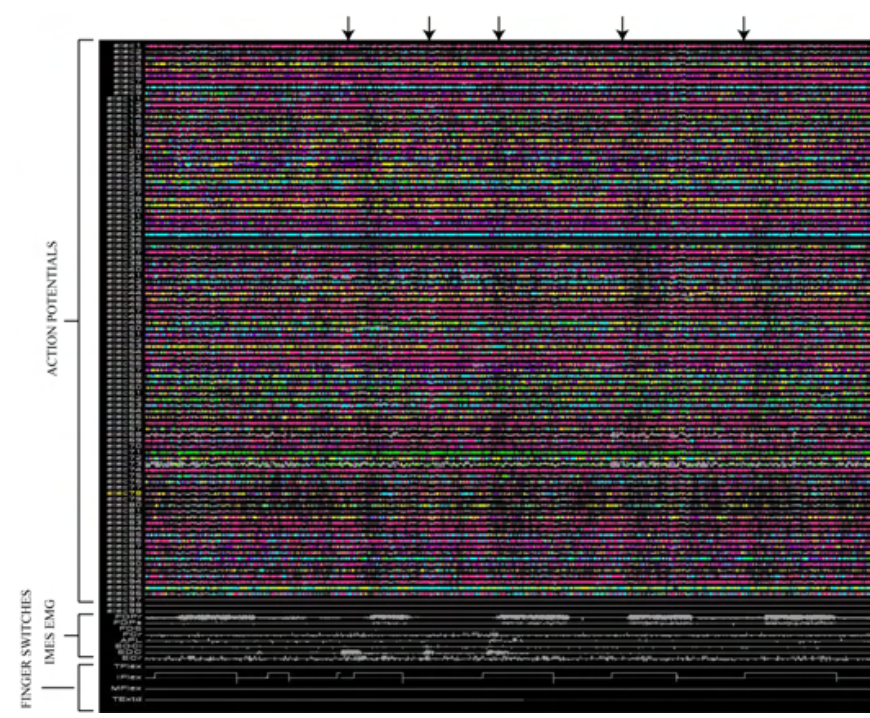

Fig. 6. Raster plot showing APs on UEA, EMG from IMES, and switch closures as the monkey performs the finger task. Arrows indicate AP modulation across the UEA and EMG modulation near finger switch closures.
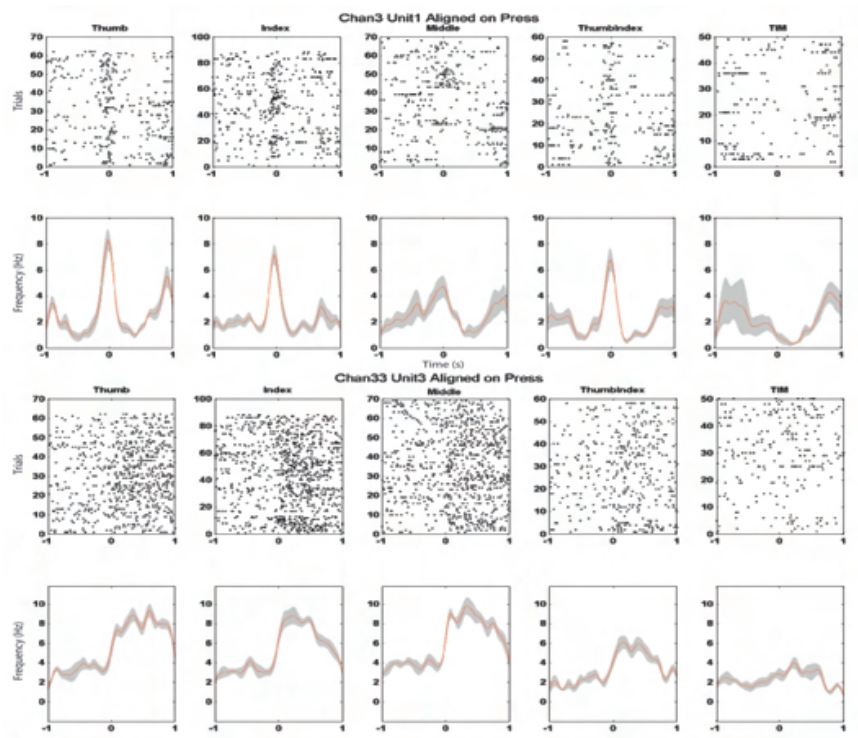

Fig. 7. Raster and PSTH plots of Unit 1 on Channel 3 and Unit 3 on Channel 33 aligned on thumb, index finger, and middle finger flexion as well as Thumb-Index and Thumb-Index-Middle (TIM) combined flexions. Notice that Channel 3 Unit 1 has a higher firing rate for thumb flexion, index flexion, and thumb-index combined flexion while the firing rate is low for movements involving the middle finger. Note the increase in firing rate after the movement for Channel 33 Unit 3.

finger switch press for the different finger movements. The average firing rate was determined by convolving the spiketimes with a Gaussian window of $\sigma=50 \mathrm{~ms}$ over all trials present for that specific finger movement. The average firing rates were then used to create peristimulus time histograms (PSTHs) for each finger movement (see Fig. 7). The PSTHs and further spectral analyses of LFP and $\mu \mathrm{ECoG}$ were performed using the Chronux toolbox (http://chronux.org [8]).

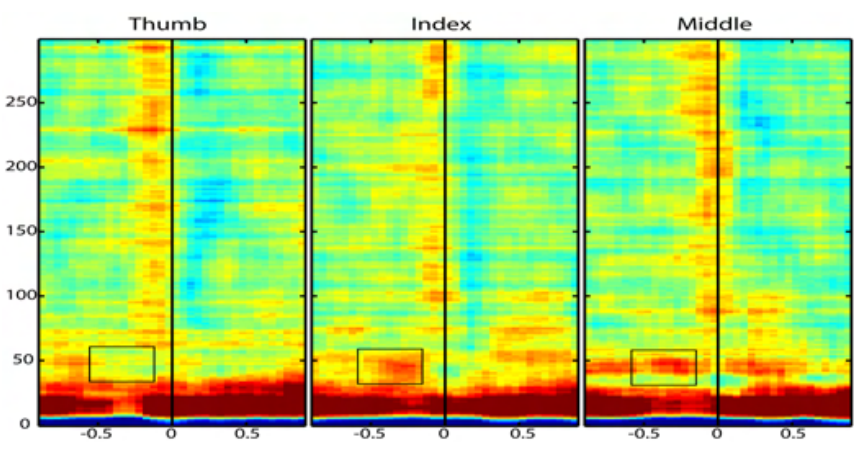

Fig. 8. Normalized trial-averaged spectrogram for LFP on electrode 63 in the UEA centered on finger flexion (thumb, index finger, and middle finger) flexion (the center black line). Note the difference in power in the gamma band just prior to finger flexion. $n=24$ thumb, 25 index, and 22 middle finger flexion trials. Time-frequency boxes indicate areas of greatest difference.

\section{$L F P$ and $\mu E C o G$ Spectrograms}

We calculated the average LFP and $\mu \mathrm{ECoG}$ spectrum across trials for a one second window during the Wait for Cue relaxation period prior to each trial to establish the baseline spectral power. We then calculated the trial-averaged spectrograms for the LFP on each electrode in the UEA and for data from the $\mu \mathrm{ECoG}$ grid centered on the finger switch closure. This baseline spectrum was then subtracted from the spectrogram to create a normalized spectrogram to better show the power changes from baseline. We compared the normalized spectrograms for the different finger movements. These normalized trial-averaged spectrograms show differences between different finger movements. This indicates that the LFP and $\mu \mathrm{ECoG}$ data may contain sufficient information to decode which finger movement is being performed (see Figs. 8, 9). The ability to decode dexterous finger movements from LFP during a similar behavioral task has been shown previously by Mollazadeh et. al. [9] and LFP selectivity for grasp has been shown by Spinks et. al. and Asher et. al. [10] [11]. However, the ability to decode dexterous finger movements using $\mu \mathrm{ECoG}$ signals has yet to be shown. The percent difference in average gamma band power $(30$ to $100 \mathrm{~Hz}$ ) over the UEA and $\mu \mathrm{ECoG}$ grid in a $300 \mathrm{~ms}$ window beginning 100 ms prior to the finger switch press was computed between the different individual finger movements (see Figs. 10, 11). This figure shows that there may be enough difference between the various finger movements that a decode could potentially be written to determine which finger was flexed based on the average power in the gamma band.

\section{Real-time Finger Flexion Decode}

We wrote an algorithm to decode finger movements in real time based on AP counts. The algorithm is a naïve Bayes classifier. We assumed uniform prior probabilities for each class (i.e. uniform probabilities for the monkey performing either a thumb, index finger, or middle finger flexion). We assumed that the class conditional firing rate for each unit can 


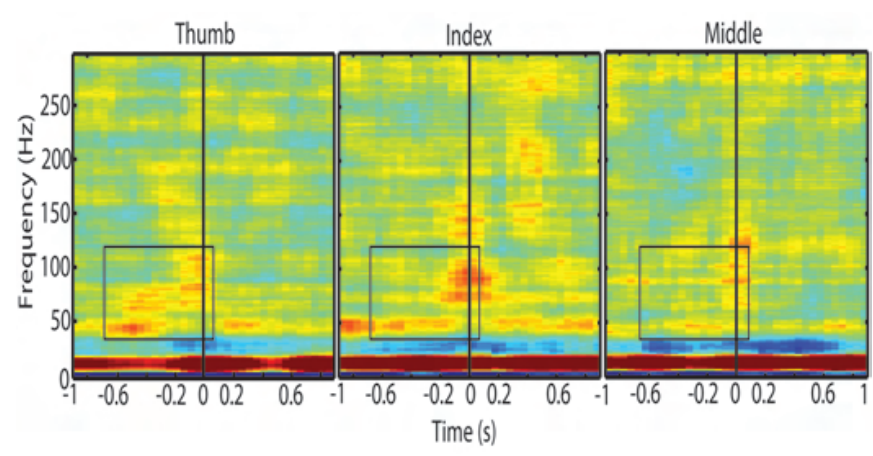

Fig. 9. Normalized trial-averaged spectrogram for electrode 13 in the $\mu \mathrm{ECoG}$ grid centered on finger flexion (thumb, index finger, and middle finger) flexion (the center black line). $n=24$ thumb, 25 index, and 21 middle finger flexion trials. Time-frequency boxes indicate areas of greatest difference.

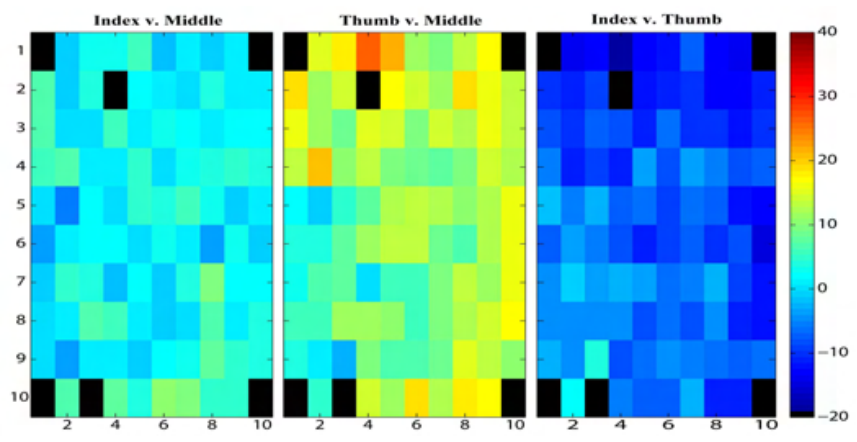

Fig. 10. Percent difference in average gamma band LFP power between the different finger movements for a $300 \mathrm{~ms}$ window beginning $100 \mathrm{~ms}$ prior to finger press on each electrode in the UEA. The same number of trials were used for each finger as in Fig. 8. Bad and reference channels blacked out.

be modeled with a Poisson distribution:

$$
P\left(n_{u} \mid \omega_{c}\right)=\frac{\lambda_{u, c}^{n_{u}} e^{-\lambda_{u, c}}}{n_{u} !}
$$

where $n_{u}$ is the observed firing rate for unit $u, \lambda_{u, c}$ is the mean firing rate for unit $u$, and $\omega_{c}$ represents class $c$. We then assume conditional independence among the firing rates for all units. This allows us to determine the likelihood of observing a collection of firing rates as:

$$
P\left(\vec{O} \mid \omega_{c}\right)=\prod_{u=1}^{U}\left(\frac{\lambda_{u, c}^{n_{u}} e^{-\lambda_{u, c}}}{n_{u} !}\right)
$$

where $\vec{O}$ represents a vector of observed spikes and $U$ represents the total number of units. Knowing the likelihood term for an observed firing rate given each class, one can solve for the posterior probability, which is the probability of each class having occurred given the observed firing rate, using Bayes' Theorem.

$$
P\left(\omega_{c} \mid \vec{O}\right)=\frac{P\left(\vec{O} \mid \omega_{c}\right) P\left(\omega_{c}\right)}{P(\vec{O})}
$$

Because we assumed equal prior probabilities, $P\left(\omega_{c}\right)$, and $P(\vec{O})$ is a constant that normalizes the posterior probabilities for all classes, the algorithm chooses to perform the movement

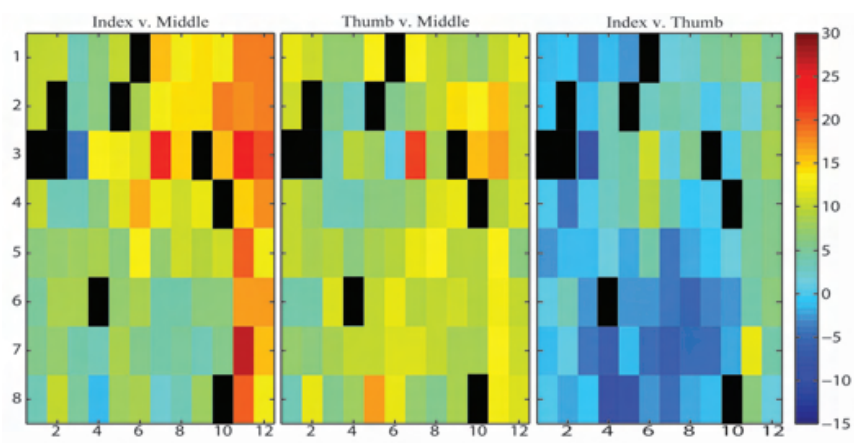

Fig. 11. Percent difference in average gamma band power between the different finger movements for a $300 \mathrm{~ms}$ window beginning $100 \mathrm{~ms}$ prior to finger press on each electrode in the $\mu \mathrm{ECoG}$ grid. The same number of trials were used for each finger as in Fig. 9. Bad and suspect channels blacked out.

with the corresponding highest likelihood. For the real time decode, the algorithm was set to bin spikes in a $150 \mathrm{~ms}$ window that ended whenever a switch closure was detected. Thus, the switch closures acted as a trigger to tell the algorithm when to compute a decision. The corresponding switch opening was used as a stop movement cue in which the algorithm returned to the no-movement state. The algorithm was allowed to use up to 100 units and was allowed to drop out units if a one-way anova showed that the unit was not significantly tuned to any of the finger movements.

We trained the algorithm using thirty successful trials of the following three classes of movement: thumb flexion, index finger flexion, and middle finger flexion. We then ran the algorithm on incoming neural data in real-time. We recorded the performance of the algorithm for four minutes. The algorithm performance over this period was $100 \%$ on 48 successfully performed trials consisting of 15 thumb flexion trials, 18 index finger flexion trials, and 15 middle finger flexion trials presented at random. The algorithm incorrectly classified two incorrectly performed trials: a middle finger flexion classified as index flexion and an index flexion classified as middle flexion. The real-time decode performance over all finger switch closures was $96 \%$. To our knowledge, this is the first time that a real-time spike decode of individuated, dexterous finger movements has been performed.

\section{Discussion}

We've shown that a UEA with $1 \mathrm{~mm}$ long electrodes implanted in the hand region of the motor cortex can access enough neurons that are sufficiently tuned to individual finger flexions that a real-time decode can be performed with greater than 95\% accuracy [12]. The PSTHs of the UEA data showed that, in general, the firing rate of all neurons was modulated due to finger movement. It was the difference in the magnitude of the firing rate modulation between different finger movements that indicated which finger was moved. Some neurons show an increase in firing rate prior to movement, followed by a decrease in firing rate after movement, while other neurons exhibit the opposite behavior. We observed that if a neuron has a high firing rate for an individual 
finger movement, then it is likely to have a high firing rate for combined movements involving that individual finger as well. This supports work done by Georgopoulos et. al. which postulated that the motor cortex encodes finger movements based on a distributed population code [13].

The normalized spectrograms from the LFP and from the $\mu \mathrm{ECoG}$ grid showed that these data are modulated due to behavior. The power throughout the $\mu \mathrm{ECoG}$ grid maps logically to the underlying cortical areas. For example, the normalized spectrograms across the entire $\mu \mathrm{ECoG}$ grid (not shown) show that on the posterior portion of the $\mu \mathrm{ECoG}$ grid the increase in power comes after the finger press, while on the anterior portion the increase in power is more closely aligned to just before and during the finger press. This is consistent with the increased power following the finger press relating to sensory feedback following the switch press from neurons in somatosensory cortex, which lies beneath the posterior portion of the grid, while the anterior portion of the grid, which lies over primary motor cortex, shows an increase in power that is likely due to neurons encoding movement.

In conclusion, the high accuracy real-time decode based on APs as well as initial trial-averaged differences among different finger movements in LFP and $\mu \mathrm{ECoG}$ spectrograms indicate that multi-scale recordings may be able to yield high accuracy decodes of finger movements from different levels of invasiveness. Our future work will be to look at the variance in the LFP and $\mu \mathrm{ECoG}$ spectrograms to determine if a real-time decode based on single trial spectral information is feasible and practical. Finally, we will use the different signals synergistically in an attempt to write more accurate and robust decodes for neuroprosthetic control of finger movements.

\section{REFERENCES}

[1] M. H. Schieber, "Individuated finger movements of rhesus monkeys: a means of quantifying the independence of the digits," J Neurophysiol, vol. 65, no. 6, pp. 1381-91, 1991.

[2] J. J. Baker, D. Yatsenko, J. F. Schorsch, G. A. DeMichele, P. R. Troyk, D. T. Hutchinson, R. F. Weir, G. Clark, and B. Greger, "Decoding individuated finger flexions with implantable myoelectric sensors," Conf Proc IEEE Eng Med Biol Soc, vol. 2008, pp. 193-196, 2008.

[3] P. A. House, J. D. MacDonald, P. A. Tresco, and R. A. Normann, "Acute microelectrode array implantation into human neocortex: preliminary technique and histological considerations." Neurosurg Focus, vol. 20, no. 5, p. E4, 2006.

[4] R. Weir, P. R. Troyk, G. DeMichele, D. A. Kerns, J. F. Schorsch, and H. Maas, "Implantable yoelectric sensors (IMESs) for intramuscular electromyogram recording," IEEE Trans Biomed Eng, vol. 56, no. 1, 2009.

[5] R. Weir, P. Troyk, G. Demichele, and D. Kerns, "Technical details of the implantable myoelectric sensor (IMES) system for multifunction prosthesis control," Conf Proc IEEE Eng Med Biol Soc, vol. 7, pp. 7337-40, 2005.

[6] P. R. Troyk, G. A. DeMichele, D. A. Kerns, and R. Weir, "IMES: an implantable myoelectric sensor," Conf Proc IEEE Eng Med Biol Soc, vol. 2007, pp. 1730-3, 2007.

[7] G. A. DeMichele, P. R. Troyk, D. A. Kerns, and R. Weir, "An implantable myoelectric sensor based prosthesis control system," Conf Proc IEEE Eng Med Biol Soc, vol. 1, pp. 2970-3, 2006.

[8] P. Mitra and H. Bokil, Observed Brain Dynamics. Oxford University Press, 2008.

[9] M. Mollazadeh, V. Aggarwal, G. Singhal, A. Law, A. Davidson, M. Schieber, and N. Thakor, "Spectral modulation of LFP activity in $\mathrm{m} 1$ during dexterous finger movements," Conf Proc IEEE Eng Med Bio Soc, vol. 2008, pp. 5314-5317, 2008 .

[10] R. Spinks, A. Kraskov, T. Brochier, M. A. Umilta, and R. N. Lemon, "Selectivity for grasp in local field potential and single neuron activity recorded simultaneously from M1 and F5 in the awake macaque monkey," J Neurosci, vol. 28, no. 43, pp. 10961-10971, 2008.

[11] I. Asher, E. Stark, M. Abeles, and Y. Prut, "Comparison of direction and object selectivity of local field potentials and single units in macaque posterior parietal cortex during prehension," J Neurophysiol, vol. 97, pp. 3684-3695, 2007.

[12] S. Acharya, F. Tenore, V. Aggarwal, R. Etienne-Cummings, M. H. Schieber, and N. Thakor, "Decoding individuated finger movements using volume-constrained neuronal ensembles in the M1 hand area," IEEE Trans Neural Sys Rehab Eng, vol. 16, no. 1, pp. 15-23, 2008.

[13] A. Georgopoulos, G. Pellizzer, A. Poliakov, and M. H. Schieber, "Neural coding of finger and wrist movements," Journal of Computational Neuroscience, vol. 6, pp. 279-288, 1999. 